\title{
Immune impairment thresholds in HIV infection
}

\author{
Shingo Iwami ${ }^{a}$, Shinji Nakaoka ${ }^{b, c}$, Yasuhiro Takeuchi ${ }^{a *}$, \\ Yoshiharu Miura ${ }^{d, e}$, Tomoyuki Miura ${ }^{f}$ \\ Graduate School of Science and Technology, Shizuoka University, Japan ${ }^{a}$, \\ Aihara Complexity Modelling Project, ERATO, JST, The University of Tokyo, Japan ${ }^{b}$, \\ Graduate School of Mathematical Sciences, The University of Tokyo, Japan ${ }^{c}$, \\ Laboratory of Viral Pathogenesis, Institute for Virus Research, Kyoto University, Japan ${ }^{d}$, \\ Department of Neurology, Saitama Prefectural Rehabilitation Center, Japan ${ }^{e}$, \\ Laboratory of Primate Model, Institute for Virus Research, Kyoto University, Japan ${ }^{f}$
}

Running title: Impairment thresholds

Total number of abstract: 235 words

Total number of text: 3158 words

Keywords: HIV infection; Dendritic cell; Immune impairment; Immunodeficiency; Distribution of asymptomatic period; Mathematical model

Corresponding author: Yasuhiro Takeuchi,

Professor of Science and Technology, Shizuoka University,

e-mail: takeuchi@sys.eng.shizuoka.ac.jp,

mailing address: 3-5-1 Johoku Naka-ku Hamamatsu 432-8561 Japan,

Tel \& Fax: +81-53-478-1200 


\section{Summary}

Longitudinal studies of patients infected with HIV-1 reveal a long and variable length of asymptomatic phase between infection and development of AIDS. Some HIV infected patients are still asymptomatic after fifteen or more years of infection but some patients develop AIDS within two years. The mechanistic basis of the disease progression has remained obscure but many researchers have been trying to explain it. For example, the possible importance of viral diversity for the disease progression and the development of AIDS has been very well worked out in the early 1990s, especially by some important works of Martin A. Nowak. These studies can give an elegant explanation for a variability of asymptomatic phase. Here, a simple mathematical model was used to propose a new explanation for a variable length of asymptomatic phase. The main idea is that the immune impairment rate increases over the HIV infection. Our model suggested the existence of so-called "Risky threshold" and "Immunodeficiency threshold" on the impairment rate. The former implies that immune system may collapse when the impairment rate of HIV exceeds the threshold value. The latter implies that immune system always collapses when the impairment rate exceeds the value. We found that the length of asymptomatic phase is determined stochastically between these threshold values depending on the virological and immunological states. Furthermore, we investigated a distribution of the length of asymptomatic phase and a survival rate of the immune responses in one HIV patient. 


\section{Introduction}

The infection with HIV-1 is characterized by various patterns of the disease progression among the patients [18, 49]. Longitudinal studies of the patients reveal a long and variable length of latency (asymptomatic) phase between infection and development of AIDS [3, 13, 28, 45]. Some HIV infected patients are still asymptomatic after fifteen or more years of infection but some patients develop AIDS within two years $[4,27]$ (the similar disease progression patterns are also observed in SIV/SHIV infected individuals [2, 21]). Although the mechanistic basis of the disease progression has remained obscure, several factors such as viral reproductive abilities and immune proliferative abilities are considered as a cause of the diverse disease progression [1, 10, 11, 14, 15, 19, 29, 47].

Above all, the possible importance of viral diversity for the disease progression and the development of AIDS have been very well worked out in the early 1990s, especially by some important works of Martin A. Nowak [29, 30, 31, 32, 33]. These very interesting studies revealed the potential existence of viral diversity threshold which states that the viral load explodes when the diversity of virus strains exceeds this threshold number. Although the diversity threshold theory can give an elegant explanation for variability of asymptomatic phase, there are several unverificational and skeptical points over HIV/SIV/SHIV disease progression (for example, the difficulty of detection of the number of quasispecies, the disease progression without increasing the viral diversity, and the limitation of viral diversity are included), and therefore it might be difficult to validate this theory by experiments.

Here we construct a simple mathematical model describing the interactions between HIV and immune cells and propose a new explanation for the variable length of asymptomatic phase in HIV infection. The main idea is that immune impairment effect increases over the HIV infection. This idea is naturally justified by progressive decrease of dendritic cell (DC) number and function, which are crucial in generation and regulation of adaptive immunity such as cytotoxic T lymphocytes (CTLs), during the course of HIV infection [8, 9, 23, 42, 43, 48].

Our model suggests the existence of two thresholds: one is "Risky threshold" which states that immune system may collapse when the impairment rate of HIV exceeds the threshold value (a risk of immunodeficiency) and the other is "Immunodeficiency threshold" which states that immune system always collapses when the impairment rate exceeds the value (a fatality of immunodeficiency). We found that the length of asymptomatic phase is determined stochastically between these threshold values depending on the virological and immunological states. Furthermore, we 
investigated a distribution of the length of asymptomatic phase and a survival rate of the immune responses in one HIV patient.

\section{Methods}

After encountering HIV in the peripheral blood, DCs mature and travel to lymphoid organs $[8,18]$. The matured DCs present HIV on MHC class II to prime CD4 ${ }^{+} \mathrm{T}$ cells to become activated $\mathrm{CD} 4^{+} \mathrm{T}$ cells which can secrete helper signals (e.g. IL2) $[14,18]$. Furthermore, the DCs cross-present HIV on MHC class I to prime $\mathrm{CD}^{+} \mathrm{T}$ cells. By receiving antigen signal from the DCs and helper signal from activated $\mathrm{CD} 4^{+} \mathrm{T}$ cells, $\mathrm{CD} 8^{+} \mathrm{T}$ cells expand and differentiate into CTLs to kill HIV infected cells $[18,26,41,49]$. Here we use a simple mathematical model describing the interactions between HIV and immune cells and explain about our modeling approach, basic assumptions, and scenario in detail.

\subsection{Dynamics of HIV replication and CTL inducement}

As described in $[5,44]$, we only consider activated $\mathrm{CD} 4^{+} \mathrm{T}$ cells $(x)$ as target cells for HIV. This is because other cell populations bearing CD4 molecule such as naive $\mathrm{CD}^{+} \mathrm{T}$ cells, resting $\mathrm{CD} 4^{+} \mathrm{T}$ cells and macrophages are less commonly infected by HIV compared with activated $\mathrm{CD}^{+} \mathrm{T}$ cells $[5,14,44]$. We assume that activated $\mathrm{CD}^{+} \mathrm{T}$ cells are produced at a rate of $\lambda$ cells day $^{-1}$, decay at a rate $d$ day $^{-1}[5,44]$, and can become infected at a rate proportional to the number of infected CD4 ${ }^{+} \mathrm{T}$ cells $(y)$ with transmission rate constant $\beta$ day $^{-1}$ cell $^{-1}[17,47]$. The infected CD $4^{+}$ $\mathrm{T}$ cells are assumed to decay at a rate $a$ day $^{-1}$.

In order to suppress HIV replication, our immune system induces CTL responses through the interactions with DCs. Here we consider that CTL responses $(z)$ include both CTL effector and memory (CTL naives are assumed to be described by initial value of $z$ ). The CTL responses eliminate infected $\mathrm{CD}^{+} \mathrm{T}$ cells at a rate proportional to the number of CTLs with killing rate constant $p$ day $^{-1}$ cell $^{-1}$ and decay at a rate $b$ day $^{-1}[17,47]$. We simply assume that proliferation of CTLs (i.e., expansion and differentiation) requires both antigen and helper signal [18, 26, 41, 49] and its rate is $c$ day $^{-1}$ cell $^{-2}$. This implies that, in the absence of any immune impairment effects, the proliferation is described by cxyz [1,36, 47]. However, in the presence of immune impairment effects caused by HIV infection, CTL proliferation is reduced (we give a detailed explanation in later). Therefore, we model immune impairment effects as $c x y z /(1+\varepsilon y)$. Here we mention that the impairment effect depends on 
the number of infected $\mathrm{CD} 4^{+} \mathrm{T}$ cells and $\varepsilon$ represents immune impairment rate.

\subsection{Mathematical model}

We extend the standard virus-immune model [29] including the effect of immune impairment caused by HIV infection. Our mathematical model is given by the following equations:

$$
\begin{aligned}
x^{\prime} & =\lambda-d x-\beta x y, \\
y^{\prime} & =\beta x y-a y-p y z, \\
z^{\prime} & =\frac{c x y z}{1+\varepsilon y}-b z .
\end{aligned}
$$

Because free virus populations decay at much faster rates than the other $\mathrm{T}$ cells, we can assume that the virus population is in a quasi steady state [46], and the dynamics of free virus is neglected in model (1) [17, 47].

\subsection{Immune impairment effect over HIV infection}

Several DC populations are targets for $\operatorname{HIV}[14,22,48]$ and, as a consequence, the ability of DCs (e.g. antigen presentation) to stimulate T cell proliferation is impaired because of depletion and dysfunction of DC (e.g. downregulation of CD80/86 and MHC II expression) $[8,9,20,22,23,35]$. The evidence comes from observations that DCs express CD4 and chemokine receptors and are susceptible to HIV infection in vitro $[35,48]$. Actually, progressive depletion of DC and its functional defects, which would in turn impair generation of CTLs, in patients with HIV-1 infection are observed $[8,9,23]$ and the reduction of DC number and function is particularly marked in patients with AIDS compared with asymptomatic subjects [23, 35]. Therefore, the progressive change of DC might contribute to the immunodeficiency associated with HIV-1 disease $[8,9,20,22,23,35,48]$. In order to understand how the progressive depletion and functional defect of DCs affect disease symptoms, we simply assume that the immune impairment rate $(\varepsilon)$ gradually increases and therefore CTL proliferation decreases over the HIV infection.

\subsection{Pathological scenario}

Usually, after infection of HIV, the virus establishes the acute infection [29]. This implies that basic reproduction number of the virus, $R_{0}=\lambda \beta / a d$, is greater than 1. Furthermore, at the end of the acute infection, CTL responses are induced and the infected cells are regulated by them at a virological set point $[18,26]$. That is, 
most of patients progress to asymptomatic phase which follows the acute phase and remain at this phase for a long time, which represents a typical disease progression of HIV infection.

Actually, if the viral infectivity (i.e., infection rate of HIV: $\beta$ ) is remarkably large, in the sense that $\lambda c / b<\beta$, our immune system do not establish CTL responses in spite of very high viral load. Because too much $\mathrm{CD} 4^{+} \mathrm{T}$ cells are destroyed during the acute infection, infected individuals immediately develop immunodeficiency without the asymptomatic phase, which represents a rapid disease progression observed in experimental SIV/SHIV infection [2, 21].

On the other hand, if the cytopathogenicity is not small, in the sense that $\lambda \beta / d-$ $\beta \sqrt{\lambda c b \beta} / c d<a$, CTL responses are hardly or not observed because of lack of antigen signals. These phenomena are considered as CTL non-responsiveness which is different from immunodeficiency of HIV infection [47] (AIDS is characterized by high virus load of HIV and CD4 ${ }^{+}$T cell depletion [18]). The CTL non-responsiveness might be observed in a viral infection with a high degree of cytopathogenicity but can not be applied to HIV infection.

Therefore, because we focus on the typical HIV disease progression in this paper, we assume that

$$
1<R_{0}, \quad \beta<\frac{\lambda c}{b}, \quad 0<a<\frac{\lambda \beta}{d}-\frac{\beta \sqrt{\lambda c b \beta}}{c d} .
$$

The detailed mathematical explanation for the derivation of the above thresholds is given in [16].

\section{Results}

We investigate how loss of DC number and function (i.e., increase of immune impairment rate) correlates with the development of immunodeficiency and discuss with a distribution of the length of asymptomatic phase.

\subsection{Risky and immunodeficiency thresholds}

We demonstrate the existence of immune impairment thresholds which can determine patients' symptoms.

Because we assumed a relatively small infection rate $(\beta)$ and cytopathogenicity of HIV $(a)$, sustained CTL responses are established and therefore the viral replication is suppressed at a low level in "controlled state" $E_{c}^{+}=\left(x_{c}^{+}, y_{c}^{+}, z_{c}^{+}\right)$after the acute infection. Actually our model has another possible interior equilibrium 
$E_{c}^{-}=\left(x_{c}^{-}, y_{c}^{-}, z_{c}^{-}\right)$but $E_{c}^{-}$is not biologically appropriate because the equilibrium is always unstable even if it exists [16].

However, as immune impairment rate increases, the CTL responses gradually become weak and the infected individuals eventually develop immunodeficiency (i.e., AIDS) because of depletion of $\mathrm{CD}^{+} \mathrm{T}$ cells (Fig.1). This progressive immune decline corresponds to the typical disease progression of HIV infection [18]. Over the disease progression, we have the following two immune impairment thresholds which can characterize the development of AIDS:

$$
\bar{\varepsilon}=\frac{a c}{b \beta}+\frac{a \beta}{a d-\lambda \beta}, \quad \varepsilon_{-}=\frac{(\sqrt{b \beta}-\sqrt{\lambda c})^{2}}{b d} .
$$

Until the impairment rate exceeds $\bar{\varepsilon}$, CTLs control the virus load at very low level, but if the impairment rate exceeds this threshold value, the patient has a risk of development of AIDS (Fig.1). This is because, in this case, "immunodeficiency state" $E_{u}=\left(x_{u}, y_{u}, 0\right)$ is stable, in which a complete breakdown of our immune system occurs (i.e., $z$ converges to 0 ), and both the controlled and immunodeficiency states can be stable simultaneously. Here we call $\bar{\varepsilon}$ "Risky threshold".

Furthermore, once the impairment rate exceeds $\varepsilon_{-}$, our immune system completely collapses, CTL responses become inactivated and the patient always develops AIDS (Fig.1). This is because the controlled equilibrium is degenerated but the immunodeficiency equilibrium remains stable. We call $\varepsilon_{-}$"Immunodeficiency threshold".

\subsection{Development of AIDS}

The development of AIDS in the patient might be affected by stochastic perturbations caused by virological and immunological events, such as mutational changes of viral epitopes and their specific immune responses [25, 26, 29], a switch of coreceptor from CCR5 to CXCR4 [18, 37], and an emergence of drug resistance [38]. Here we investigate an impact of the stochastic perturbations on a deterioration of the disease (Fig.2).

Usually, virus load of HIV equilibrates and remains at a virological set point just after the acute infection $[18,26]$. Even if the immune impairment rate increases, the viral replication is well controlled by CTL responses at the controlled state until the rate exceeds the risky threshold (i.e., $0<\varepsilon<\bar{\varepsilon}$ ). This is because only $E_{c}^{+}$is stable, and therefore the immune suppression is robust for any stochastic perturbation (Fig.2). 
However, when the impairment rate exceeds the risky threshold (i.e., $\bar{\varepsilon}<\varepsilon$ ), the stochastic perturbations lead to the development of immunodeficiency (Fig.2) because of the bistability of controlled state and immunodeficiency state. Furthermore, to investigate a progressive risk of immunodeficiency, we plot a basin of attraction (i.e., absorbing region) of $E_{u}$ as red region which is called "Risky zone" in Fig.3 (if their virological and immunological states are located on the risky zone, an HIV patient eventually develops immunodeficiency). Because the risky zone gradually expands during the impairment rate increases (simulations are not shown) until the immunodeficiency threshold $\left(\varepsilon<\varepsilon_{-}\right)$, the patient becomes sensitive to these stochastic perturbations (i.e., the risk of the development of immunodeficiency is progressively increased).

In addition, once the impairment rate exceeds the immunodeficiency threshold $\left(\varepsilon_{-}<\varepsilon\right)$, the risky zone expands into total space (simulations are not shown) and the patient always develops AIDS because the immunodeficiency state $E_{u}$ becomes unique stable steady state.

Thus, the development of AIDS (i.e., the length of asymptomatic phase) is determined stochastically between these threshold values depending on the virological and immunological states (Fig.2).

\subsection{Distribution of length of asymptomatic phase}

It is a well-known empirical fact that incubation times of most diseases obey a lognormal distribution that is often referred to as 'Sartwel's model' [19, 40]. For example, the waiting times between HIV infection and the development of AIDS is considered as a lognormal distribution. We mention that these results are based on cross-sectionally epidemiological studies of the HIV patients from around the world $[3,13,45]$. Actually, it is poorly understood how the incubation times (i.e., the possibility of the development of AIDS) are distributed in one HIV patient. Here we study the distribution of the length of asymptomatic phase (i.e., a probability density function of development of AIDS) based on within host dynamics (1) in one HIV patient which shows the typical disease progression.

We calculate 1000 trials of time-course of the disease progression in the context of stochastic perturbations and investigate frequency of the development of immunodeficiency at each immune impairment rate in Fig.4. The histogram in Fig.4 represents a distribution of the length of asymptomatic phase (the median values $\varepsilon=986.61$ ). Here we simply assume that the length of asymptomatic phase is represented by the immune impairment rate at which CTL responses vanish. The blue curve drawn by solid line in Fig.4 represents a survival rate of the immune responses (i.e., the 
probability that an HIV patient has not yet developed AIDS at each impairment rate). The two dashed-curves represent a $95 \%$ confidence interval of the survival rate.

Because the patient does not develop AIDS until the impairment rate exceeds the risky threshold, the distribution (or the median value) leans to the range of large impairment rate and the survival curve is flat in the range of small impairment rate. However, because the patient has a (stochastic) risk of development of AIDS once the rate exceeds the risky threshold and the risk is progressively increased until the immunodeficiency threshold, the distribution tends to have a center peak and the survival curve begins to decay gradually. Furthermore, because the patient always develops AIDS after the impairment rate exceeds the second threshold, the shape of the distribution is characterized by a short right tail and the survival rate vanishes.

Thus, we obtain the relatively wide shape of the distribution of the length of asymptomatic phase between the risky thresholds and immunodeficiency thresholds and a gradually decreasing function of the survival rate of the immune responses. These results predict the existence of long and variable asymptomatic phase between infection and development of AIDS.

\section{Discussion}

The origin of various disease progression in HIV infection is largely unresolved but many researchers have been trying to explain it. An important factor in understanding the unusual incubation period distribution in the development of AIDS is the dynamics of the long-lasting struggle between HIV and our immune system [19]. Until now, several approaches have been proposed to explain the long-term fight in HIV infection $[1,10,11,14,15,19,29,47]$. For example, in very early models of HIV infection worked by Martin A. Nowak, increasing of HIV variants' diversity, which leads to an explosion in the virus load, explained the collapse of immune system after a long incubation period. They claimed the possible importance of viral diversity for the disease progression and the development of AIDS [29, 30, 31, 32, 33].

Here we discussed about an immune impairment effect caused by the depletion and dysfunction of DC on HIV disease progression. Because the progressive decrease of DC number and function during the course of HIV-1 infection is observed [8, 9, 23 , we simply assumed that the immune impairment rate $(\varepsilon)$ increases over the HIV infection instead of directly considering a population dynamics of DCs. In the typical disease progression which is characterized by relatively small infection rate $(\beta)$ and cytopathogenicity of $\operatorname{HIV}(a)$, we demonstrated that increasing of 
immune impairment rate eventually leads to the development of immunodeficiency and proposed the possibility of the existence of the risky threshold $(\bar{\varepsilon})$ and the immunodeficiency threshold $\left(\varepsilon_{-}\right)$over the disease progression (Fig.1 and 2). Our theoretical framework can explain the disease progression as many experimenters hypothesized that the progressive alteration of the immune system (the depletion and dysfunction of DCs are central to many of these hypotheses) might be caused by the development of AIDS [8, 9, 20, 22, 23, 35, 42, 48].

Using our within host dynamics model (1), we also investigated a distribution of the length of asymptomatic phase for a typical disease progressor in Fig.4. From cross-sectionally epidemiological studies of the HIV patients from around the world, it is said that the distribution among the patients is considered as a lognormal distribution $[3,13,45]$ (e.g. a cellular automaton model with a sequence space framework reported that the incubation time is a lognormal-like distribution [19]). However, our predictable distribution based on the within host dynamics in one HIV patient is different from the well-known distribution obtained by the cross-sectional studies (actually, it is difficult to reveal the distribution in one HIV patient from epidemiological data). The shape of the distribution is characterized by the center peak and the short right tail. Furthermore, the right tail of the distribution might be characterized by a small peak at the end, if stochastically long-term-survival trials exist (simulations are not shown), because the patient always develops AIDS above the immunodeficiency threshold. Thus, our theoretical framework leads to an epidemiologically-falsifiable (or -difficult) predictions which merit further experimental investigations in SIV/SHIV infection.

In summary, we found that increasing of the immune impairment rate caused by the progressive decrease of DC number and function plays an important role in HIV infection. This implies that recovering DC function and increasing DC number are effective in inducing immune response and delaying the disease progression (actually, several studies of DC immunotherapy found transient decrease of the virus load by activating $\mathrm{T}$ cell responses against HIV-1 $[6,12,39])$ because the modulation of DC leads to decrease of the immune impairment rate. Furthermore, if the impairment rate can be quantified by time-course data of DCs $[8,9,23]$, our theory might enable to predict a real-time risk of the development of AIDS for each patients.

Acknowledgments We would like to thank anonymous referees and the editor for very helpful suggestions and comments which improved the quality of this paper and study. 
Funding SI was supported by Research Fellowships of the Japan Society for the Promotion of Science for Young Scientists and SN was supported by (i) Research Fellowships of the Japan Society for the Promotion of Science for Young Scientists and (ii) the Sasakawa Scientific Research Grant from The Japan Science Society.

Author Contributions All authors conceived the model and analyzed the model. SI wrote the paper.

Competing Interests The authors have no competing interests.

\section{References}

[1] Altes HK, Wodarz D,Jansen VAA. The dual role of CD4 T helper cells in the infection dynamics of HIV and their importance for vaccination. J. Theor. Biol. 2002; 214:633-646.

[2] Binley JM, Clas B, Gettie A, Vesanen M, Montefiori DC, Sawyer L, et al. Passive infusion of immune serum into simian immunodeficiency virus-infected rhesus macaques undergoing a rapid disease course has minimal effect on plasma viremia. Virology 2000; 270:237-249.

[3] CASCADE Collaboration.Time from HIV-1 seroconversion to AIDS and death before widespread use of highlyactive antiretroviral therapy: a collaborative re-analysis. Lancet 2000; 355:1131-1137.

[4] Cecilia D, Kleeberger C, Munoz A, Giorgi JV, Zolla-Pazner S. A longitudinal study of neutralizing antibodies and disease progression in HIV-1-infected subjects. J. Infect. Dis. 1999; 179:1365-1374.

[5] Ciupe MS, Bivort BL, Bortz DM, Nelson PW. Estimating kinetic parameters from HIV primary infection data through the eyes of three different mathematical models. Math. Biosc. 2006; 200:1-27.

[6] Connolly NC, Whiteside TL, Wilson C, Kondragunta V, Rinaldo CR, Riddler SA. Therapeutic immunization with human immunodeficiency virus type 1 (HIV-1) peptide-loaded dendritic cells is safe and induces immunogenicity in HIV-1-infected individuals. Clin. Vaccine. Immunol. 2008; 15:284-292. 
[7] Davenport MP, Ribeiro RM, Perelson AS. Kinetics of virus-specific CD8+ T cells and the control of human immunodeficiency virus infection. J. Virol. 2004; 78:10096-100103.

[8] Donaghy H, Pozniak A. Gazzard B, Qazi N, Gilmour J, Gotch F, et al. Loss of blood CD11c+ myeloid and CD11c- plasmacytoid dendritic cells in patients with HIV-1 infection correlates with HIV-1 RNA virus load. Blood 2001; 98:2574-2576.

[9] Donaghy H, Gazzard B, Gotch F, Patterson S. Dysfunction and infection of freshly isolated blood myeloid and plasmacytoid dendritic cells in patients infected with HIV-1. Blood 2003; 101:4505-4511.

[10] de Boer RJ, Boerlijst MC. Diversity and virulence thresholds in AIDS. Proc. Nat. Acad. Sci. U.S.A. 1994; 94:544-548.

[11] Galvani AP. The role of mutation accumulation in HIV progression. Proc. Roy. Soc. Lond. B 2005; 272:1851-1858.

[12] Garcia F, Lejeune M, Climent N, Gil C, Alcami J, Morente V, et al. Therapeutic immunization with dendritic cells loaded with heat-inactivated autologous HIV1 in patients with chronic HIV-1 infection. J. Infect. Dis. 2005; 191:1680-1685.

[13] Gayet-Ageron A, Baratin D, Marceillac E, Allard R, Peyramond D, Chidiac C, et al. The AIDS epidemic in Lyon: patient characteristics and defining illnesses between 1985 and 2000. HIV Med. 2004; 5:163-170.

[14] Hogue IB, Bajaria SH, Fallert BA, Qin S,Reinhart TA, Kirschner DE. The dual role of dendritic cells in the immune response to human immunodeficiency virus type 1 infection. J. Gen. Virol. 2008; 89:2228-2239.

[15] Iwami S, Nakaoka S, Takeuchi Y. Viral diversity limits immune diversity in asymptomatic phase of HIV infection. Theor. Pop. Biol. 2008; 73:332-341.

[16] Iwami S,Miura T, Nakaoka S, Takeuchi Y. Immune impairment effect in HIV infection: Existence of risky and immunodeficiency thresholds. In Revision.

[17] Iwasa Y, Michor F, Nowak MA. Virus evolution within patients increases pathogenicity. J. Theor. Biol. 2005; 232:17-26.

[18] Janewa C, Travers P, Walport M, Shlomchik MJ. Immunobiology: The immune system in health and disease. Garland Pub. 2004. 
[19] Kamp C, Bornholdt S. From HIV infection to AIDS: a dynamically induced percolation transition? Proc. R. Soc. Lond. B 2002; 269:2035-2040.

[20] Kawamura T, Gatanaga H, Borris DL, Connors M, Mitsuya H, Blauvelt A. Decreased stimulation of CD4+ T cell proliferation and IL-2 production by highly enriched populations of HIV-infected dendritic cells. J. Immunol. 2003; 170:4260-4266.

[21] Kozyrev IL, Ibuki K, Shimada T, Kuwata T, Takemura T,Hayami M, et al. Characterization of less pathogenic infectious molecular clones derived from acute-pathogenic SHIV-89.6P stock virus. Virology 2001; 282:6-13.

[22] Lore K, Sonnerborga A, Brostrom C, Goh L-E, Perrin L, McDade H, et al. Accumulation of DC-SIGN+CD40+ dendritic cells with reduced CD80 and CD86 expression in lymphoid tissue during acute HIV-1 infection. AIDS 2002; 16:683-692.

[23] Macatonia SE, Lau R, Patterson S, Pinching AJ, Knight SC. Dendritic cell infection, depletion and dysfunction in HIV-infected individuals. Immunology 1990; 71:38-45.

[24] Mandl JN, Regoes RR, Garber DA, Feinberg MB. Estimating the effectiveness of simian immunodeficiency virus-specific CD8 $+\mathrm{T}$ cells from the dynamics of viral immune escape. J. Virol. 2007; 81:11982-11991.

[25] McKnight A, Clapham PR. Immune escape and tropism of HIV. Tre. Micro. 1995; 3:356-361.

[26] McMichael AJ, Rowland-Jones SL. Cellular immune responses to HIV. Nature $2001 ; 410: 980-987$.

[27] Meissnera EG, Duus KM, Gao F, Yu X-F, Su L. Characterization of a thymustropic HIV-1 isolate from a rapid progressor: role of the envelope. Virology 2004; 328:74-88.

[28] Mellors JW. Viral-load tests provide valuable answers, Scient. Amer. 1998; July:70.

[29] Nowak MA, May RM. Virus dynamics. Oxford University Press, 2000.

[30] Nowak MA. Evolutionary dynamics. Harvard University Press, 2006. 
[31] Nowak MA, Anderson RM, McLean AR, Wolfs TF, Goudsmit J, May RM. Antigen diversity thresholds and the development of AIDS. Science 1991; 15:963-969.

[32] Nowak MA. Variability in HIV infections. J. Theor Biol. 1992; 155:1-20.

[33] Nowak MA, May RM. AIDS pathogenesis: mathematical models of HIV and SIV infection. AIDS 1993; 7:S3-S18.

[34] Ogg GS, Jin X, Bonhoeffer S, Moss P, Nowak MA, Monard S, et al. Decay kinetics of human immunodeficiency virus-specific effector cytotoxic T lymphocytes after combination antiretroviral therapy. J. Virol. 1999; 73:797-800.

[35] Patterson S, English NR, Longhurst H, Balfe P, Helbert M, Pinching AJ, et al. Analysis of human immunodeficiency virus type 1 (HIV-1) variants and levels of infection in dendritic and T cells from symptomatic HIV-1-infected patients. J. Gen. Virol. 1998; 79:247-257.

[36] Regoes RR, Wodarz D, Nowak M A. Virus dynamics: the effect of target cell limitation and immune responses on virus evolution. J. Theor. Biol. 1998; 191:451-462.

[37] Regoes RR, Bonhoeffer S. The HIV coreceptor switch: a population dynamical perspective. Tre. Micro. 2005; 13:269-277.

[38] Richman DD. HIV chemotherapy. Nature 2001; 410:995-1001.

[39] Rinaldo CR. Dendritic cell-based human immunodeficiency virus vaccine. J. Int. Med. 2008; 265:138-158.

[40] Sartwell P. The incubation period and the dynamics of infectious disease. Am. J. Epidemiol. 1966; 83:204-216.

[41] Schmitz JE, Kuroda MJ, Santra S, Sasseville VG, Simon MA, Lifton MA, et al. Control of viremia in simian immunodeficiency virus infection by CD8 $(+)$ lymphocytes. Science 1999; 283:857-860.

[42] Smed-Sorensen A, Lore K, Walther-Jallow L,Anderson J, Spetz A. HIV-1 infected dendritic cells up-regulate cell surface markers but fail to produce IL-12 p70 in response to CD40 ligand stimulation. Blood 2004; 104:2810-2817.

[43] Soumelis V, Scott I, Gheyas F, Bouhour D, Cozon G, Cotte L,et al. Depletion of circulating natural type 1 interferon-producing cells in HIV-infected AIDS patients. Blood 2001; 98:906-912. 
[44] Stafford MA, Corey L, Cao Y, Daar ES, Ho DD, Perelson AS. Modeling plasma virus concentration during primary HIV infection. J. Theor. Biol. 2000; 203:285301.

[45] Tassie J-M, Grabar S, Lancar R, Deloumeaux J, Bentata M, Costagliola D, et al. Time to AIDS From 1992 to 1999 in HIV-1-Infected Subjects With Known Date of Infection. J. AIDS 2002; 30:81-87.

[46] Thieme HR. Mathematics in population biology. Princeton University Press, 2003.

[47] Wodarz D, Klenerman P, Nowak MA. Dynamics of cytotoxic T-lymphocyte exhaustion. Proc. Roy. Soc. Lond. B 1998; 265:191-203.

[48] Wu L, KewalRamani V N. Dendritic-cell interactions with HIV: infection and viral dissemination. Nat. Rev. Immunol. 2006; 6:859-868.

[49] Tunetsugu-Yokota Y. How does HIV infection destory the host immune system? J. AIDS Reser. 2005; 7:171-179. 

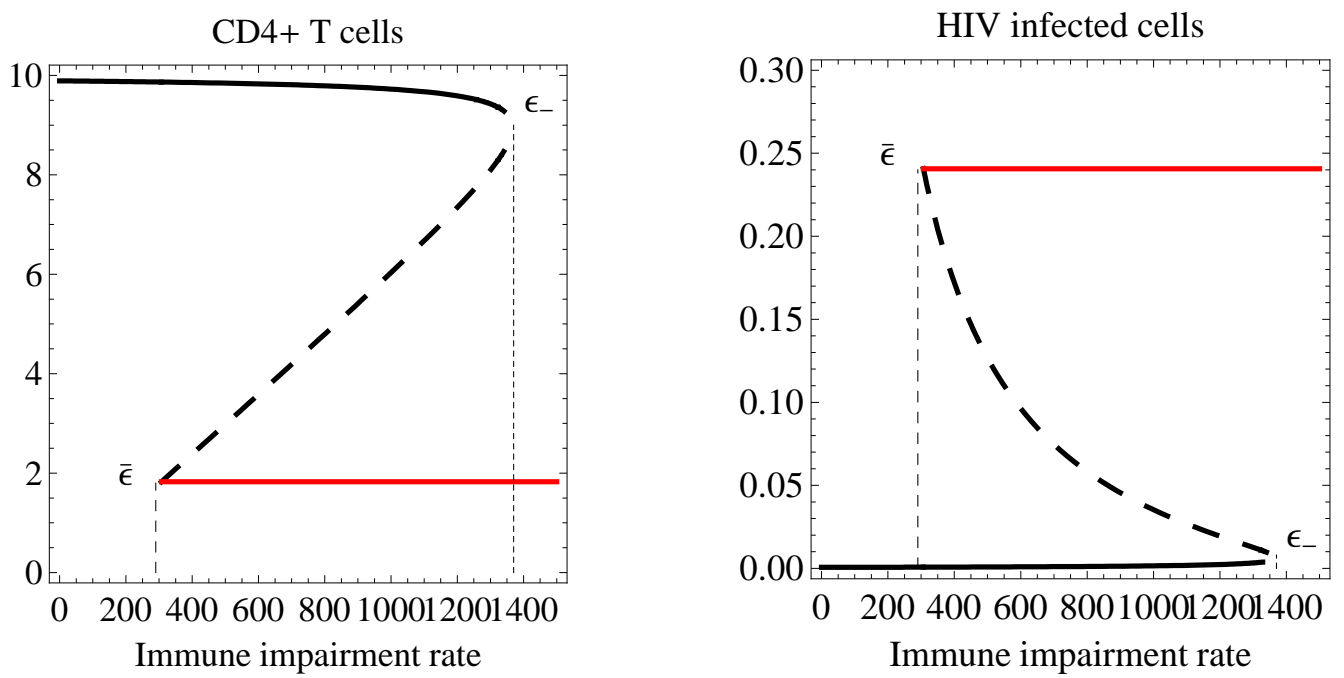

CTL responses

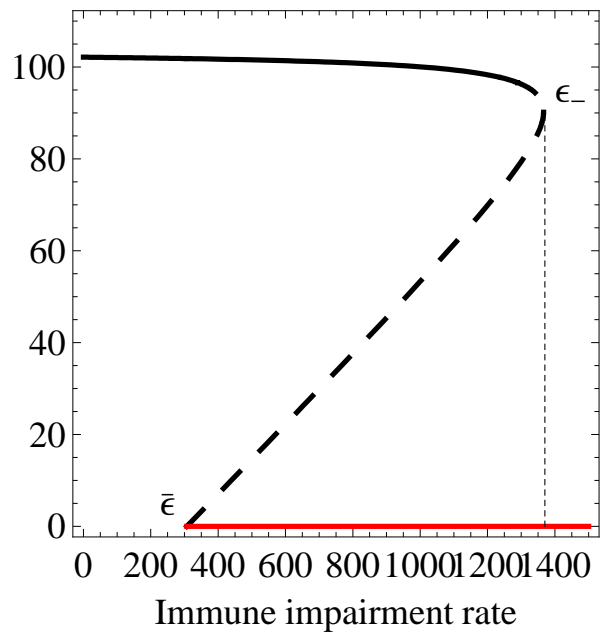

Figure 1: Typical disease progression in HIV infection: the black and red solid lines, respectively, represent each cell number at the controlled and immunodeficiency state over HIV infection (the black dashed lines represent each cell number at $E_{c}^{-}$). Because the immune impairment rate is small at the beginning of the infection, the sustained CTLs suppress HIV replication. However, the CTL responses gradually decrease and the infected individuals become to have a risk of development of AIDS, if the impairment rate exceeds the risky threshold (i.e., $\bar{\varepsilon}<\varepsilon$ ), and they always develop AIDS, if the rate exceeds the immunodeficiency threshold (i.e., $\varepsilon_{-}<\varepsilon$ ). Here we choose our baseline parameter values as follows: $\lambda=0.1089, d=0.01089$, $\beta=0.2027, a=0.366, p=0.016, c=2.13$, and $b=0.0125$. These values are based on previously estimated parameter values in [7, 24, 34, 44]. Because we are interested in change of immune impairment effect and their threshold phenomena, we set $\varepsilon$ a free parameter. The detailed explanation of the estimate of our baseline parameter values is given in [16]. 


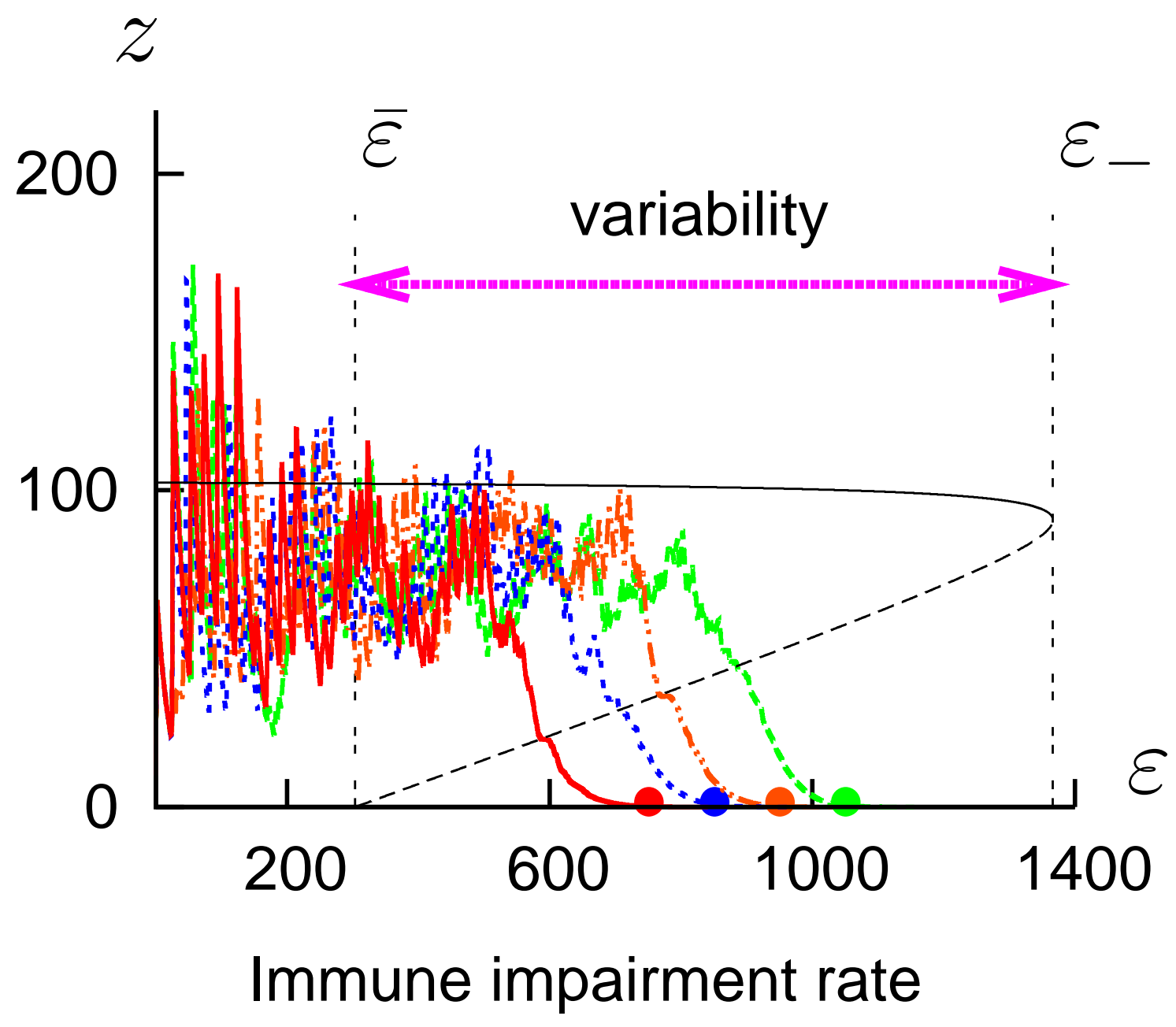

Figure 2: Development of AIDS caused by stochastic perturbations: we calculate time-course of the disease progression in the context of stochastic perturbations.

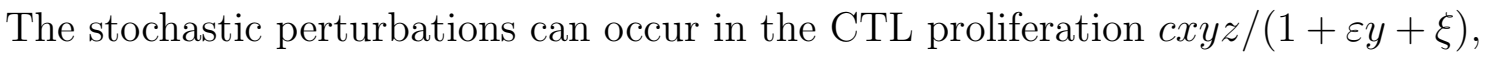
where $\xi$ is a noise measured by a lognormal distribution. We assume that, because of progressive depletion and dysfunction of DC, the immune impairment accumulates with a very low rate during disease progression, $\varepsilon(t)=0.25 t$, where 0.25 is the rate of average impairment per day. The most severe patient (red line) develops AIDS after the impairment rate exceeds around 700. However, the most mild patient (green line) do not develop AIDS until the rate exceeds around 1000. The development of AIDS is determined stochastically between these threshold values depending on the virological and immunological states. Therefore, we can explain the variable length of asymptomatic phase in HIV infection. Here we use the same parameter values in Fig.1. The risky and immunodeficiency thresholds, respectively, are estimated to $\bar{\varepsilon}=303.63$ and $\varepsilon_{-}=1366.45$. 


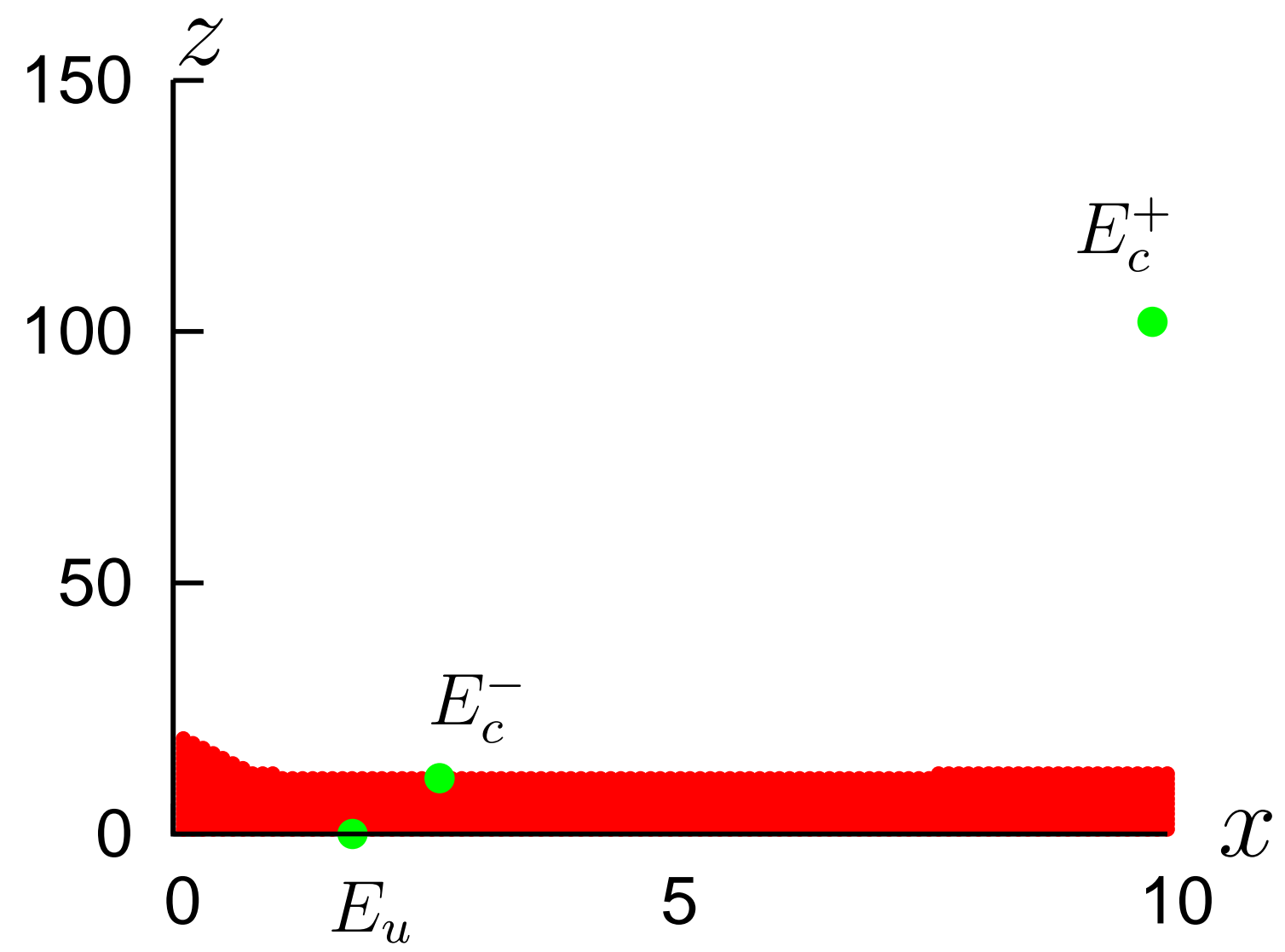

Figure 3: Risky zone of immunodeficiency: we plot a basin of attraction of $E_{u}$ as red region and call it "Risky zone". Until the immune impairment rate $(\varepsilon)$ exceeds the risky threshold $(\bar{\varepsilon})$, only $E_{c}^{+}$is stable. On the other hand, when the impairment rate exceeds the risky threshold, $E_{c}^{-}$is generated into $\mathbb{R}_{+}^{3}$ via a transcritical bifurcation with $E_{u}$ and the risky zone emerges (i.e, both $E_{c}^{+}$and $E_{u}$ become stable simultaneously). The stable manifold of $E_{c}^{-}$forms boundary surface of the risky zone. And also, the risky zone expands during the impairment rate increases until the immunodeficiency threshold $\left(\varepsilon_{-}\right)$. However, once the impairment rate exceeds the immunodeficiency threshold, $E_{c}^{ \pm}$are degenerated via a saddle-node bifurcation and full space becomes the risky zone (i.e, only $E_{u}$ is stable). Here we use the same parameter values in Fig.1 and set $\varepsilon=450$. 

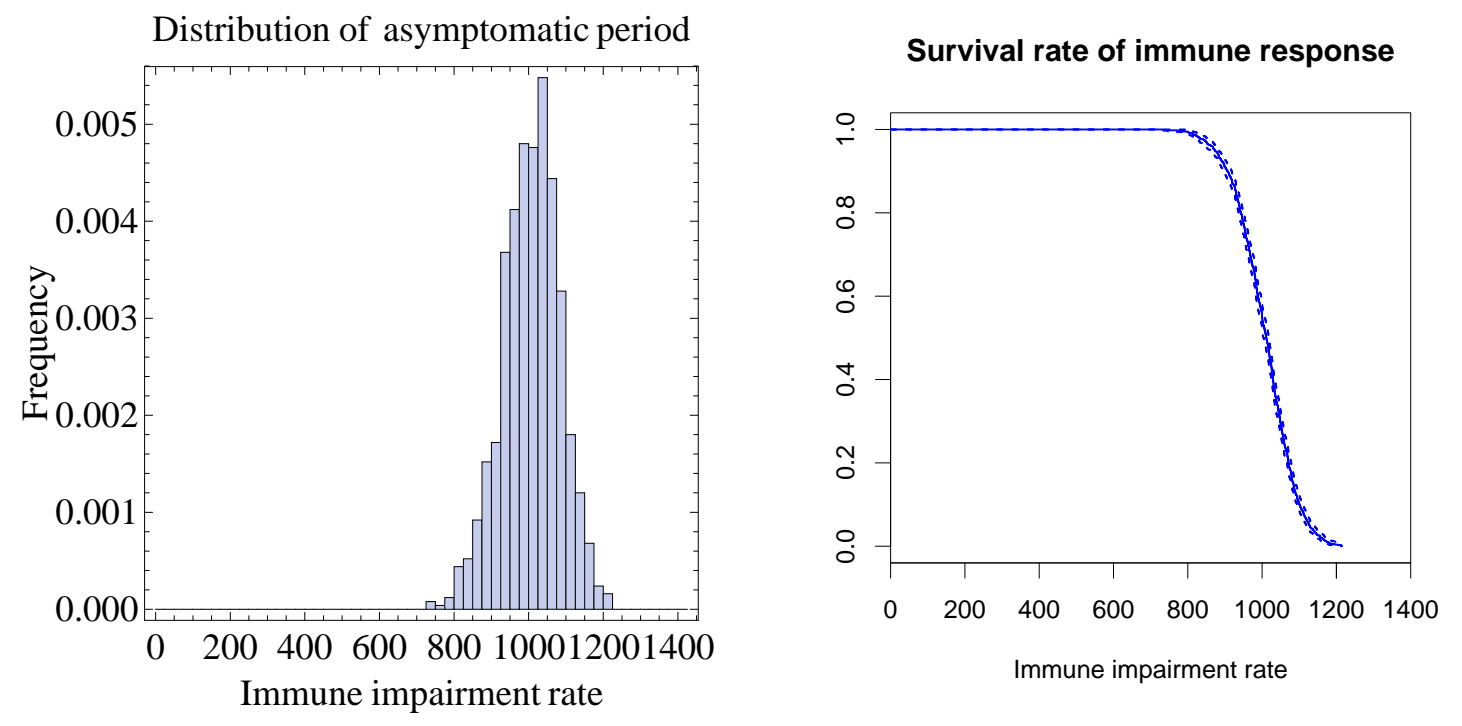

Figure 4: Distribution of length of asymptomatic phase: we calculate 1000 trials of time-course of the disease progression in the context of stochastic perturbations (we use the same method in Fig.2) and investigate frequency of the development of immunodeficiency at each immune impairment rate. The left histogram represents a distribution of the length of asymptomatic phase (here we simply consider that the length of asymptomatic phase is represented by the immune impairment rate at which CTL responses vanish). We assume that the categories of the histogram are 60 and the scale of the histogram is 1 (i.e., each category represents a probability density). The right function drawn by solid blue line represents a survival rate of the immune responses (i.e., the probability that an HIV patient has not yet developed AIDS at each impairment rate). The two dashed-lines represent a 95 $\%$ confidence interval of the survival rate. Estimation for the distribution of the survival rate is computed with GNU R package for survival analysis. These results imply the existence of long and variable asymptomatic phase between infection and development of AIDS. Here we use the same parameter values in Fig.1. The risky and immunodeficiency thresholds, respectively, are estimated to $\bar{\varepsilon}=303.63$ and $\varepsilon_{-}=1366.45$. 